\title{
Cavitary Lung Lesions, Rash, Systemic Shock, Hematuria, and Acute Kidney Injury in a Patient with Elevated Anti-Nuclear Antibody Titers and Rheumatoid Factor
}

\author{
Mehret Birru Talabi1 1 , Douglas Lienesch¹, Kimberly Liang1
}

${ }^{1}$ University of Pittsburgh and University of Pittsburgh Medical Center

\begin{abstract}
We present a case of a 42 year-old male with history of complicated gastric bypass and recent deep vein thrombosis who presented with purpuric rash, systemic shock, and cavitary lung lesions, and leukocytosis, acute kidney injury, and elevated RF, anti-nuclear antibody titer, and inflammatory markers. Initial presentation was concerning for systemic vasculitis. Aspiration pneumonia with resultant lung abscesses was ultimately diagnosed. No corticosteroids or immunosuppression were initiated at any point during the management of the patient, and he improved with antibiotics and drainage of the abscesses. Cavitary lung lesions are rare in rheumatic diseases, which helped to narrow the differential diagnosis. Mimics of vasculitis include malignancy and infection, and often pose a diagnostic and therapeutic challenge.
\end{abstract}

Keywords: Vasculitis, Cavitary Lung Lesion, Leukocytoclastic Vasculitis, Pigmented Purpuric Dermatosis

\section{Presentation of Case}

A 42 year-old Caucasian male with a history of Roux-en-Y gastric bypass, iron deficiency anemia with chronic indwelling port for iron transfusions, and recent deep vein thrombosis (DVT), was in his usual state of health until 3 days prior to presentation, when he discovered a small, painless, erythematous papule on his left calf. Over the next few days, the calf became edematous and indurated. He sought care at a local emergency department and was diagnosed with cellulitis. Cephalexin was prescribed, but soon after initiating treatment, his entire left calf became increasingly erythematous and edematous, and the rash spread to his right calf. He also developed progressive fatigue and malaise.

He presented back to the local emergency department. Vital signs indicated normal temperature and oxygen saturation on room air, but he was tachycardic to 120 beats per minute and hypotensive with blood pressure of 70/40. Initial laboratory evaluation was remarkable for white blood cell count of $36,400 \times 10 \mathrm{e} 9$ with $95.5 \%$ neutrophils and no eosinophils, platelets of $40,000 \times 10 \mathrm{e} 9$, and acute kidney injury (creatinine $4.3 \mathrm{mg} / \mathrm{dL}$, with normal baseline renal function). $\mathrm{He}$ received inotropic support and broad-spectrum antibiotics for presumed septic shock.

Computed tomography (CT) of his chest, abdomen, and pelvis revealed a new large, fluid-filled cavitary lesion at the right lung base $(10.9 \times 6.4 \mathrm{~cm})$ and a smaller cavitary lesion in the right upper lobe of his lung (Figure 1). No lymphadenopathy was noted. Transthoracic echocardiogram showed a normal ejection fraction of $60 \%$ without valvulopathy or vegetations.

Additional laboratory studies included an angiotensin-converting enzyme level of $26 \mathrm{U} / \mathrm{mL}$ (normal); negative anti-nuclear antibody (ANA); normal Complement 3 and 4 levels, negative serologies for human immunodeficiency virus (HIV), Hepatitis B and C; negative anti-Jol antibody and Scl-70 antibody. Rheumatoid factor (RF) was elevated to $33.3 \mathrm{IU} / \mathrm{mL}$ (normal $<20 \mathrm{IU} / \mathrm{mL}$ ) and ferritin was elevated to $584 \mathrm{ng} / \mathrm{mL}$ (normal 10-282 $\mathrm{ng} / \mathrm{mL}$ ). Serial blood cultures (from port and periphery), Coccidioides, Histoplasma, and Legionella testing were negative. Sputum gram stain showed more than 25 segmented WBCs and few

This article is published under the terms of the Creative Commons Attribution License 4.0

Author(s) retain the copyright of this article. Publication rights with Alkhaer Publications.

Published at: http://www.ijsciences.com/pub/issue/2015-09/

DOI: 10.18483/ijSci.829; Online ISSN: 2305-3925; Print ISSN: 2410-4477 
gram-positive cocci. Urinalysis showed 6-10 red blood cells.

Punch biopsy of the initial skin lesion on the left calf was read by an outside pathologist as "small-vessel leukocytoclastic vasculitis" (LCV).

The patient was transferred to our tertiary care institution for further evaluation and management.

\section{Past Medical History:}

The patient had a Roux-en-Y gastric bypass in 2001 complicated by recurrent anastamotic ulcers, perforated ulcer, and esophagitis; he underwent several surgical corrections, including partial gastrectomy. He developed iron-deficiency anemia post-bypass and had a chronic indwelling port implanted for periodic iron infusions. One month prior to current presentation, he had an unprovoked left femoral deep vein thrombosis (DVT). Anticoagulation was initiated with rivaroxaban.

\section{Family and Social Histories:}

No known autoimmune or connective tissue diseases were in his family. He was currently unemployed, and denied tobacco, alcohol, drug use, homelessness or incarcerations.

\section{Physical Examination:}

On physical exam, the patient was alert and oriented. Height was $175 \mathrm{~cm}$ and weight was 102 kilograms. $\mathrm{He}$ was afebrile with a respiratory rate of 22 breaths/minute, heart rate of 102 beats/minute, blood pressure of $156 / 81$, and oxygen saturation of $92 \%$ on room air. Head, eye, ear, nose, and throat examination was remarkable only for an oral ulcer on his upper palate. Respiratory examination revealed decreased breath sounds at the base of his right lung but no wheezes. No murmurs were appreciated on cardiovascular examination. Musculoskeletal examination revealed 2-3+ pitting edema in his lower extremities bilaterally without joint swelling or deformities. Integumentary examination showed non-blanching, confluent, erythematous purpuric and petechial rash, with bullous appearance or some areas, on both the right and left lower extremities, worse on the left. No fluctuance was appreciated. Neurologic examination revealed intact cranial nerves, reflexes, normal sensation and no focal weakness.

\section{Laboratory and Radiographic Evaluation:}

Additional laboratory testing at our institution revealed the following: white blood cell count had decreased to $22.7 \times 10 \mathrm{e} 9$ (91\% neutrophils) and renal function normalized $(\mathrm{Cr} 0.8 \mathrm{mg} / \mathrm{dL})$ from his initial presentation at the outside hospital. Hemoglobin was $9.0 \mathrm{~g} / \mathrm{dL}$ (baseline of $10 \mathrm{~g} / \mathrm{dL}$ ), platelets $106,000 \times 10 \mathrm{e} 9$; hepatic function tests were normal with exception of hypoalbuminemia of $2.0 \mathrm{mg} / \mathrm{dL}$. ANA was now positive at 1:320 (nucleolar pattern), rheumatoid factor (RF) was again elevated to 205 $\mathrm{mg} / \mathrm{dL}$, and CRP was high at $13.2 \mathrm{mg} / \mathrm{dL}$ (normal $<0.748)$. However, anti-cyclic citrullinated peptide (CCP), double-stranded DNA (dsDNA), SSA, SSB, Smith and RNP antibodies, anti-neutrophil cytoplasmic antibodies (ANCA) including proteinase-3 (PR3) and myeloperoxidase (MPO) antibodies, cryoglobulins, ribosomal $\mathrm{P}$, centromere, and chromatin antibodies were all negative or within normal limits. Repeat urinalysis showed trace blood, but was otherwise unremarkable. Given his recent history of unprovoked DVT, indicators of hypercoagulability were assessed: he did not meet criteria for a lupus anticoagulant, and beta-2 glycoprotein and cardiolipin antibodies were negative. Interferon gamma release testing for tuberculosis, repeat blood, viral, and fungal cultures were all negative.

Radiographs of his hands and feet showed no erosions or periarticular osteopenia.

Doppler ultrasonography of his upper and lower extremities showed no new thrombosis.

Repeat punch biopsy of the left calf rash showed dermal edema with marked erythrocyte extravasation and negative Gram stains most consistent with capillaritis associated with leg edema, a form of pigmented purpuric dermatosis (PPD). Secondary review of initial biopsy was also more consistent with PPD rather than LCV.

Repeat CT of the chest showed an increase in the size of the right pleural cavitary lesion and parenchymal collection $(11.9 \times 11 \mathrm{~cm})$ and adjacent gas locules. These findings were concerning for abscesses or fistula. The largest cavitary lesion approximated the staple line from his prior esophagojejunal anastomosis. Barium swallow and esophogastroduodenoscopy were subsequently performed, which showed no fistula between esophagus and lung but did show a marked stricture at the esophagojejunal anastamosis.

Thoracentesis of the right pleural effusion yielded unremarkable pleural fluid, with negative cultures and cytology. Notably, thoracentesis was performed twelve days after antibiotics were started.

Aspiration pneumonia with complicated parapneumonic effusion in the setting of esophageal stricture was thereby considered the most likely diagnosis.

\section{Case Discussion}

This case involved a middle-aged male presenting with two cavitary lung lesions of unknown etiology and lower extremity petechial and purpuric skin 
lesions. Systemic vasculitis, infection, and leak from prior esophagojejunal anastomosis were the leading differential diagnoses.

A cavitary lung lesion is a nodule or consolidation with a gas-filled, radiolucent center. It is nearly always pathologic and arises from inflammation, infection, or malignancy. Certain features of this case suggested that infection was the cause of the cavitary lesions: upon initial presentation, he was in shock, disseminated intravascular coagulation, with a leukocytosis to $34,000 \times 10 \mathrm{e} 9$, and clinical improvement with broad-spectrum antibiotics. However, other features of the case suggested an autoimmune etiology of the lesions, including LCV (later reported to be PPD by another pathologist), oral ulcer, elevated ANA, RF, and inflammatory markers, kidney injury and hematuria.

Few autoimmune or connective tissue diseases are associated with cavitary lung lesions, including granulomatosis with polyangiitis (GPA), RA, and sarcoidosis. Nodules with cavitation are found in 35$50 \%$ of patients with GPA involving the lung [1], and likelihood of cavitation increases with nodule size. Cavitary lesions in GPA generally appear thickwalled with irregular margins. With treatment, approximately half resolve, $40 \%$ decrease in size, and $10 \%$ persist despite resolution of systemic symptoms [2]. Approximately $16 \%$ of GPA cavitary nodules in one study were superinfected, which underscores the complexity of differentiating between infectious and autoimmune etiologies of the lesions [1]. Our patient improved without any immunosuppression, and ANCA serologies were negative, which made GPA less likely.

Given the elevated RF, our differential diagnosis included RA-associated cavitary nodules. However, the incidence of pulmonary nodules in RA is approximately $1 \%$. As the nodules enlarge, up to $50 \%$ may cavitate, at which point they are classified as necrobiotic nodules [8]. Pulmonary vasculitis in RA is rare, and may infrequently manifest as pulmonary nodules. RA pulmonary vasculitis is associated with male gender, erosive synovitis and seropositivity; it is often accompanied by other extraarticular manifestations of disease [7]. Our patient was anti-CCP negative, and the lack of active synovitis on exam or erosive arthropathy on radiographs made the diagnosis of RA unlikely.

Atheroembolic disease may present with skin ulcerations, low serum complements and elevated inflammatory markers, which can suggest an SLEassociated vasculitis. As this patient did have elevated ANA and recent DVT, we considered systemic lupus erythematosus (SLE) and septic thromboembolic phenomena in the differential diagnosis. However, SLE-associated cavitary lesions are uncommon. One case series reported cavitary lesions in 6 of 798 SLE patients [11]. Infectious etiologies of the cavitary lesions represented 4 of the 6 cases, a pulmonary infarct comprised 1 case, and the remaining case had unknown etiology. Notably, our patient likely did not have SLE, given an absence of compatible disease features on his review of systems, and largely negative serologic workup, including fluctuating ANA results (negative at the outside institution but positive on recheck), negative dsDNA and normal complement levels. Antiphospholipid antibodies were negative, and repeat Dopplers of his lower extremities showed no new thrombosis, making septic thromboemboli less likely as well.

While sarcoidosis was not one of the leading differential diagnoses in this case, it is frequently associated with pulmonary nodules. However, the prevalence of cavitary sarcoidosis is only $2.2 \%$ [2]. A proportion of these lesions may be superinfected, most frequently with fungal organisms [4].

Infection is the most common etiology of cavitary lung lesions, and must remain high on the differential diagnosis of any cavitary lung lesion in a patient with or without underlying rheumatologic disease. Those patients on immunomodulatory therapy are at greatest risk of developing infectious cavitary lung lesions, especially with atypical organisms. Anaerobic, aerobic, or mixed bacteria, mycobacteria, filamentous organisms (Actinomyces or Nocardia), and fungal infections (particularly endemic organisms, such as Histoplasma, Coccioides, and Blastomycosis) are common inciting pathogens [5]. In this case, we were unable to identify an inciting organism. Sensitivity of cultured material was likely affected by antibiotic exposure; oral antibiotics were started initially as an outpatient, and parenteral antibiotics were started prior to culture of sputum and pleural fluids.

This case underscores that the etiology of systemic vascular damage-manifested in our patient by pulmonary, integumentary, and renal impairmentcan be challenging to elucidate. ANCA testing is sensitive for generalized GPA (80-95\%), but less so for "limited" GPA Microscopic Polyangiitis (70\%) and much less so for Churg-Strauss Syndrome (50\%); therefore, the absence of ANCA alone in our case did not definitively rule out vasculitis [10]. ANCA also has variable specificity, and infective endocarditis [10], inflammatory bowel disease, levamisole-laced cocaine [12], cardiac myxoma, in addition to other disease states are associated with elevated ANCA titers [9]. 
Vasculopathy and vasculitis may be difficult to distinguish by appearance of cutaneous lesions, including similar appearances of palpable purpura and/or petechiae in urticarial lesions, capillaritis (PPD), and LCV. Histopathologic evaluation of tissues can often help to differentiate between vasculopathy and vasculitis [6]. Notably, however, the two pathologists who reviewed the skin lesions in this case-each from a different institutiondiagnosed LCV and capillaritis (PPD), respectively. Our case highlights the diagnostic challenge experienced by many rheumatologists in determining whether a constellation of clinical findings, serologies, and other tests are consistent with vasculitis or one of its many mimics. Consideration of this patient's complicated surgical history and repeating the chest $\mathrm{CT}$ at our institution for serial comparisons helped us to reach the final diagnosis.

\section{Clinical Course:}

The patient recovered with antibiotics and drainage of the pulmonary abscess. His rash also improved shortly after the pulmonary abscess was drained. Repeat CT scan three months after discharge revealed significant decrease in the size of the cavitary lung lesions, with no new areas of cavitation.

\section{Summary Points:}

1. Cavitary lung lesions are relatively uncommon in rheumatic disorders

2. Mimics of vasculitis include infection and malignancy

\section{References:}

1. Aberle DR, Gamsu G, Lynch D. Thoracic manifestations of Wegener granulomatosis: diagnosis and course. Radiology. 174 , 703-709

(1994).
2. Brauner MW, Grenier P, Mompoint D, et al. Pulmonary sarcoidosis: evaluation with high-resolution CT. Radiology. 467-471

http://dx.doi.org/10.1148/radiology.172.2.2748828

(1989).

3. Castañer E, Alguersuari A, Gallardo X, et al. When to suspect pulmonary vasculitis: radiologic and clinical clues. $\begin{array}{llll}\text { Radiographics. } & 30(1), & 33-53 & \text { (2010). }\end{array}$ http://dx.doi.org/10.1148/rg.301095103

4. Hours S, Nunes H, Kambouchner M, et al. Pulmonary cavitary sarcoidosis: clinic-radiologic characteristics and natural history of a rare form of sarcoidosis. Medicine. 87(3), $142-51 \quad$ (2008). http://dx.doi.org/10.1097/md.0b013e3181775a73

5. Gadkowski LB, Stout JE. Cavitary Pulmonary Disease. Clin Microbiol Rev. 21(2), 305-333 (2004). http://dx.doi.org/10.1128/cmr.00060-07

6. Mahr A, Batteux F, Tubiana S, et al. Brief report: Prevalence of antineutrophil cytoplasmic antibodies in infective endocarditis. Arthritis Rheum. 66(6), 1672-7 (2014). http://dx.doi.org/10.1002/art.38389

7. Makol A, Crowson CS, Wettler DA, et al. Vasculitis associated with rheumatoid arthritis: a case-control study. Rheumatology. 53(5), $\quad 89-9 \quad$ (2014). http://dx.doi.org/10.1093/rheumatology/ket475

8. Ozkaya $S$, Bilgin S, Hamsici S, Findik S. The pulmonary radiologic findings of rheumatoid arthritis. Respiratory $\begin{array}{llll}\text { Medicine. } & 4(4), & 187-192 & \text { (2011). }\end{array}$ http://dx.doi.org/10.1016/j.rmedc.2011.03.003

9. Radice A, Bianchi L, Sinico RA. Anti-neutrophil cytoplasmic autoantibodies: methodologic aspects and clinical significance in systemic vasculitis. Autoimmun Rev. 12(4) 487-95

(2013). http://dx.doi.org/10.1016/j.autrev.2012.08.008

10. Seo P, Stone JH. The antineutrophil cytoplasmic antibodyassociated vasculitides. Am J Med. 117, 39-50 (2004). http://dx.doi.org/10.1016/j.amjmed.2004.02.030

11. Webb WR, Gamsu G. Cavitary pulmonary nodules with systemic lupus erythematosus: differential diagnosis. $A m J$ Roentgenol. $\quad 136, \quad 27-31 \quad$ (1981). http://dx.doi.org/10.2214/ajr.136.1.27

12. Wiesner O, Russell KA, Lee AS, et al. Antineutrophil cytoplasmic antibodies reacting with human neutrophil elastase as a diagnostic marker for cocaine-induced midline destructive lesions but not autoimmune vasculitis. Arthritis Rheum. 50(9), 2954-65 (2004). http://dx.doi.org/10.1002/art.20479

Fig 1.

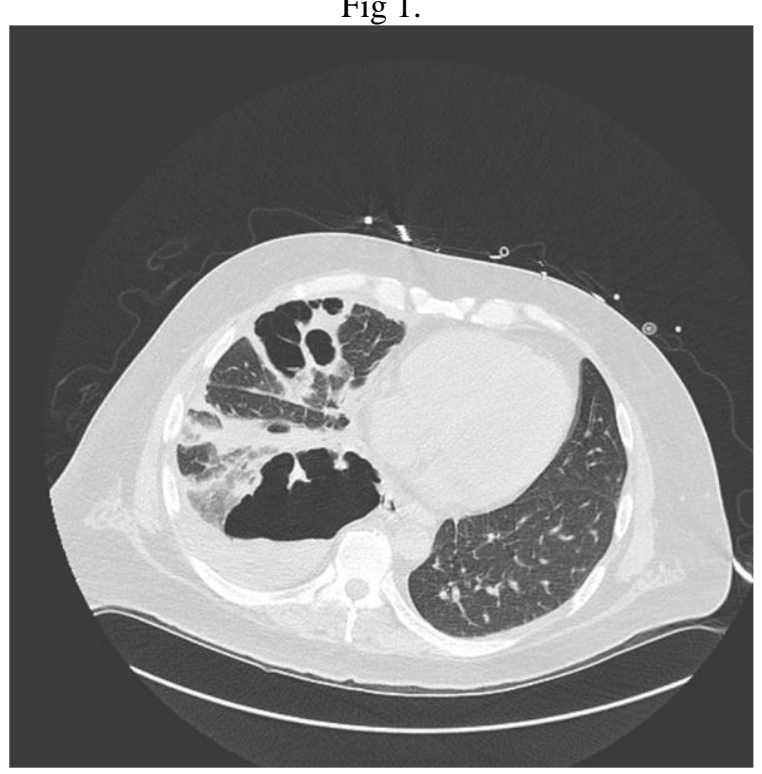

